\title{
A Review of Metamaterials and its Applications
}

\author{
Gurwinder Singh $^{\# 1}$, Rajni ${ }^{* 2}$, Anupma Marwaha ${ }^{* 3}$ \\ ${ }^{\# 1}$ Lecturer \& Corresponding author, Department of ECE, Govt. Polytechnic College, Ferozepur, Punjab, India \\ ${ }^{* 2}$ Associate Professor, Department of ECE, SBSSTC, Ferozepur, Punjab, India \\ ${ }^{*}$ Associate Professor, Department of ECE, SLIET, Sangrur, Punjab, India
}

\begin{abstract}
During the past ten years, a great interest in the research of metamaterials has been observed. Metamaterials are artificially invented materials that show the properties which are not detected in naturally occurring materials. Metamaterials exhibits negative permittivity and/ or negative permeability. This paper presents a review of different types of metamaterials, their own unique properties and advantages.
\end{abstract}

Keywords-Metamaterials(MTM), Double negative metamaterials (DNG), Negative Index Metamaterials (NIMs), Left handed Metamaterials (LHM), Split Ring Resonators (SRRs).

\section{INTRODUCTION}

The word 'Meta' is taken from Greek whose meaning is "beyond". "Metamaterials" has the exotic properties beyond the natural occurring materials. These are the materials that extract their properties from their structure rather than the material of which they are composed of. The first and one of the most important contributions to this topic was made in 1968 by V. G. Veselago who said that materials with both negative permittivity and negative permeability are theoretically possible [1]. In 1999, John Pendry identified a practical way to make left-handed metamaterials (LHM) which did not follow the conventional right hand rule [2]. He proposed his design of periodically arranged Thin-Wire (TW) structure that depicts the negative value of effective permittivity [3]. It was shown that the structure is having a low plasma frequency than the wave in the microwave regime. Because of its low plasma frequency, this structure can produce an effective negative permittivity at microwave frequencies. It was also demonstrated that negative magnetic permeability could be achieved using an array of split-ring resonators [4]. Later then, Smith demonstrated a new LHM that shows simultaneously negative permittivity and permeability and carried out microwave experiments to test its uncommon properties in 2000 [5]. Shelby et al showed negative refraction experimentally for the first time using a metamaterials with repeated unit cells of split ring resonators (SRR) and copper strips [6-7]. Wu et al proposed three structures including symmetrical ring, omega and S structure for SRRs [8]. Many researchers have worked on metamaterials to extract their potential in various fields. This paper summarizes history of metamaterials, its classification, advantages and applications.

\section{Classification of Metamaterials}

Electromagnetic field is determined by the properties of the materials involved. These properties define the macroscopic parameters permittivity $\varepsilon$ and permeability $\mu$ of materials [9] On the basis of permittivity $\varepsilon$ and permeability $\mu$, the metamaterials are classified in following four groups as shown in Fig. 1:

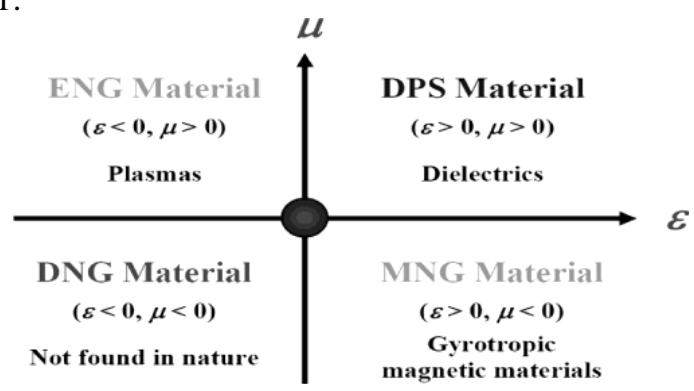

Fig.1 Classification of metamaterials

\section{A. Double Positive (DPS) Material}

The materials which have both permittivity \& permeability greater than zero $(\varepsilon>0, \mu>0)$ are called as double positive (DPS) materials. Most occurring media (e.g. dielectrics) fall under this designation.

\section{B. Epsilon Negative (ENG) Material}

If a material has permittivity less than zero and permeability greater than zero $(\varepsilon<0, \mu>0)$ it is called as epsilon negative (ENG) material. In certain frequency regimes, many plasmas exhibit these characteristics.

\section{Mu Negative(MNG)Material}

If a material has permittivity greater than zero \& permeability less than zero $(\varepsilon>0, \mu<0)$ it is called as mu negative (MNG) material. In certain frequency regimes, some gyro tropic material exhibits these characteristics.

\section{Double Negative (DNG) Material}

If a material has permittivity \& permeability less than zero $(\varepsilon$ $<0, \mu<0)$ it is termed as double negative (DNG) material. This class of materials can only been produced artificially.

\section{METAMATERIALS TYPES}

\section{A. Electromagnetic Metamaterials}

Electromagnetic metamaterials (EM) are the materials which have a new sub section within electromagnetism and physics. EM is used for optical and microwave applications like, band-pass filters, lenses, microwave couplers, beam steerers, and antenna radomes. 


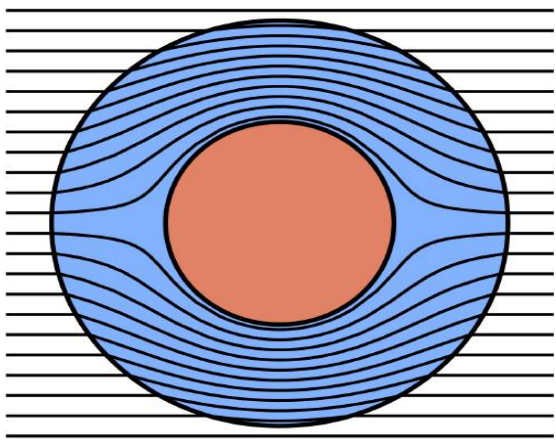

Fig.2 Electromagnetic metamaterials

A metamaterials affects lesser on electromagnetic waves as compared to wavelength of electromagnetic radiation.

1) Single Negative Metamaterials: Single negative metamaterials (SNG) have either negative permittivity or negative permeability. The combination of two SNG layers into one creates another form of DNG metamaterials [10]. To conduct wave reflection experiments, the slab of MNG materials and ENG materials have been joined. Like DNG metamaterials, SNGs change their parameters like refraction index $n$, permittivity $\varepsilon$ and permeability $\mu$, with change in frequency due to their dispersive nature.

2) Double Negative Metamaterials: Double negative metamaterials (DNG) are the metamaterials that have both permittivity and permeability is negative with negative index of refraction. These are also known as negative index metamaterials (NIM) [11]. Other names for DNGs are left handed media, media with a negative refractive index, and "backward-wave media [12].

3) Electromagnetic Band Gap (EBG) Metamaterials: Electromagnetic band gap metamaterials control the propagation of light. It is achieved either by photonic crystals (PC), or left-handed materials (LHM). Both classes have artificial structure that control and manipulate the propagation of electromagnetic waves.

4) Bi-isotropic and Bi-anisotropic Metamaterials: Based on the independent electric and magnetic responses described by the parameters permittivity and magnetic permeability, the metamaterials are categorized into single or double negative. However in many examples of electromagnetic metamaterials, the electric field causes magnetic polarization, and the magnetic field induces an electrical polarization, i.e., magneto electric coupling. Such media denoted as bi-isotropic media because it exhibits magneto-electric coupling that is anisotropic, and also called as bi-anisotropic.

\section{B. Chiral Metamaterials}

Chiral metamaterials consist of arrays of dielectric gammadions or planar metallic on a substrate. When a linearly polarized light is incident on the array, it becomes elliptically polarized upon interaction with the gammadions with the same handedness as the gammadion itself [13].

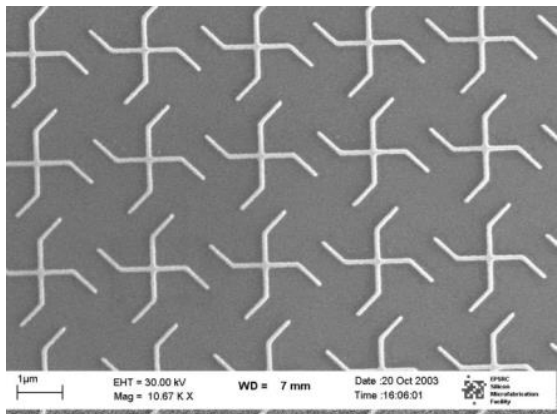

Fig.3 Chiral metamaterials

\section{Terahertz Metamaterials}

Terahertz metamaterials are the combination of artificial materials that interact at terahertz $(\mathrm{THz})$ frequencies and still under development. With negative values of permeability these metamaterials can achieve a desired magnetic response is called passive materials. Because of this, "tuning" is achieved by fabricating a new material with slightly altered dimensions to create a new response. Terahertz waves lie just before the start of the microwave band to far end of the infrared band.

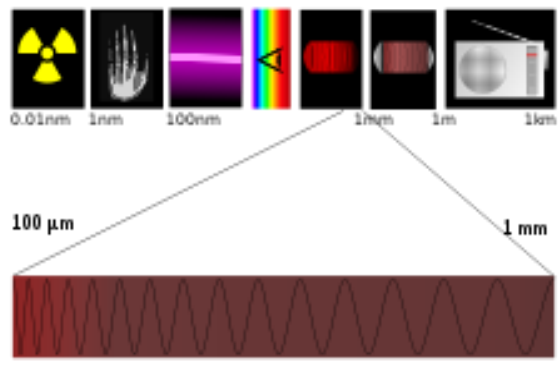

Fig.4 Wave formation of Terahertz metamaterials

The range of terahertz metamaterials frequency is 0.1 to 10 $\mathrm{THz}$ for research or other applications.

\section{Photonic Metamaterials}

Photonic metamaterials are the type of electromagnetic metamaterials that designed to interact with optical frequencies is known as Optical metamaterials. Photonic metamaterials radiates the source at optical wavelengths [14]. Furthermore, the sub wavelength period differentiates the photonic metamaterials from photonic band gap structure. This is because the optical properties do not arise from photonic band gaps, rather from a sub wavelength interaction with the light spectrum. The metamaterials with the capability of zero index of refraction (ZIMs) and negative values for index of refraction (NIMs) is the active area of research in optical materials. 


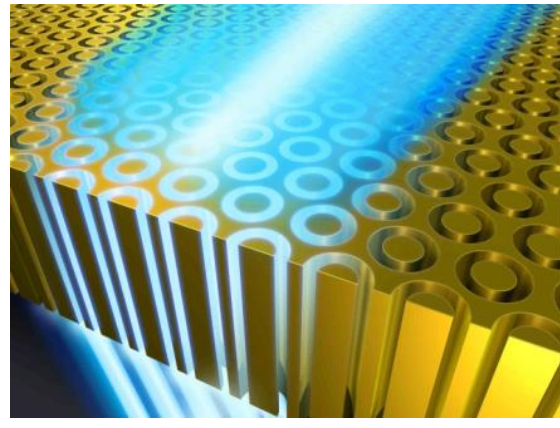

Fig.5 Photonic Metamaterials

\section{E. Tunable Metamaterials}

These are the metamaterials that has the ability to randomly change the frequency of a refractive index. An incident electromagnetic wave gives variable response with these metamaterials. This includes how an incident electromagnetic wave interacts with a metamaterials in remote controlling. The structure of the tunable metamaterials is changeable in real time that makes it possible to reconfigure a device during operation [15]. Tuning in the near infrared range is achieved by varying the permittivity of nematic liquid crystal. The metamaterials can be tuned from negative index values, to zero index or positive index values. In addition, negative index values can be increased or decreased.

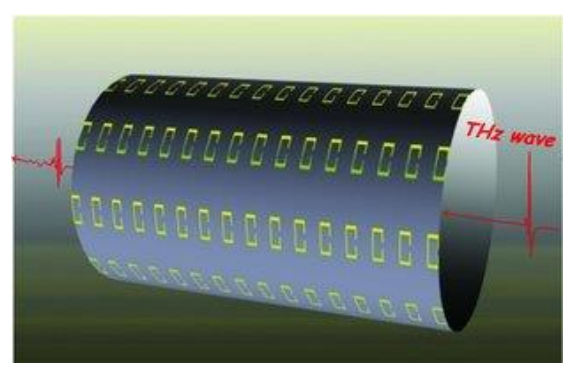

Fig.6 Tunable Metamaterials

\section{F. Frequency Selective Surface (FSS) based Metamaterials}

FSS based metamaterials are the substitute to the fixed frequency metamaterials with static geometry and spacing in the unit cells used to find out the frequency response of a given metamaterials. FSS based metamaterials have option to change the frequencies in a single medium but in fixed frequency response it is impossible. It was first developed to control the transmission and reflection characteristics of an incident radiation wave. FSS with specific geometrical shapes can be made-up as periodic arrays with elements of two dimensional planar. FSS based metamaterials has the interchangeable terminology of High Impedance Surface (HIS) or Artificial Magnetic Conductor (AMC). The HIS or AMC has an artificial metallic electromagnetic structure. The designed structures with selection of supporting surface wave currents are different from conservative metallic conductors.

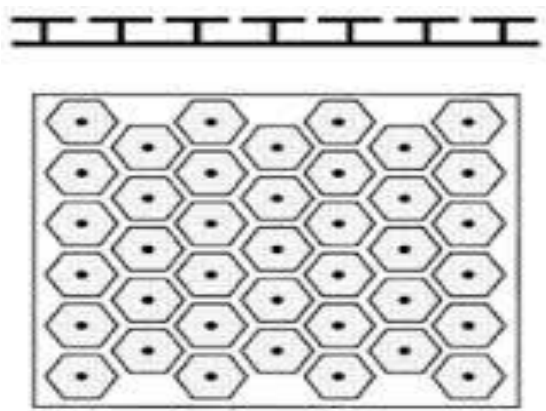

Fig.7 Structure of FSS

\section{G. Nonlinear Metamaterials}

Nonlinear metamaterials are artificial materials in which the nonlinearity exists. This is due to less macroscopic electric field of the electromagnetic source than the microscopic electric field of the inclusions [15]. The material's permeability and permittivity describes the response of electromagnetic radiation. It may also be fabricated with some type of nonlinear metamaterials that have properties to change the power of incident wave.

\section{METAMATERIALS APPLICATIONS}

Metamaterials applications are found in various fields including public safety, sensor detection, high frequency battle field communication, improving ultrasonic sensors, solar power management, for high gain antennas and remote aerospace applications [16-17].

\section{A. WMD Detectors}

Army and air Force researcher use metamaterials to detect the presence of chemical explosives, biological agents, and contamination. The same structure and science is used for cargo and screening passenger.

\section{B. Invisible Subs}

The metamaterials easily manipulate the wavelength of sound that is much larger than light. To make the submarine invisible to enemy sonar, it bends sound around it and this program is funding by Naval Research. Civilian spinoffs also use the metamaterials to produce sound proofing rooms with perfect acoustics.

\section{Revolutionary Electronics}

Army engineers use the metamaterials as a switching device for building fast and small photonic equipment because in future circuits makes with the use of light rather than electricity. To trap light can be turned on and off a semiconductor combines with the metamaterials in the device. These photonic chips are 10 times faster than the current chips.

\section{Light and sound filtering}

To control light or sound signals that improve ultrasound resolution and change a material's color, nano scale wrinkles of metamaterials are used. These materials can be made with a multi-layer deposition process and high-precision. By using a 
fraction of a wavelength each of the layer's thickness can be control. The precise wrinkles spacing scatters the selected frequencies which created by compressing the materials [1819]. These are used in medical diagnostics, sound suppression and nondestructive material testing.

\section{E. Biosensor}

Biosensors are very important tools in many areas, like environmental monitoring, food safety and disease diagnostics in the investigation of biological phenomena's [20-21]. In past the fluorescence-based methods were used to prove it but recently metamaterials been used by researchers in bio sensing technologies because of its detection of label-free bio molecules and efficient cost [22-23].

\section{F. Metamaterials Absorber}

A metamaterial absorber efficiently absorbs electromagnetic radiation. Metamaterials absorbers offer benefits over conventional absorbers such as supplementary miniaturization, wider adaptability, and increased effectiveness [24]. To create a high ratio of electromagnetic radiation absorption, it requires effective medium design, permittivity and magnetic permeability.

\section{G. Metamaterial Antennas}

Metamaterials are used in antennas to increase performance of miniaturized antenna systems [25-26]. The metamaterials antennas are use to increase the gain of an antenna because it has a unique band gap features and periodic structures [27]. In small conventional antennas the most of the wavelength reflects the signal back to the source. But the metamaterials antenna has structure that stores and re-radiates energy which makes its size small and behaves as larger antenna.

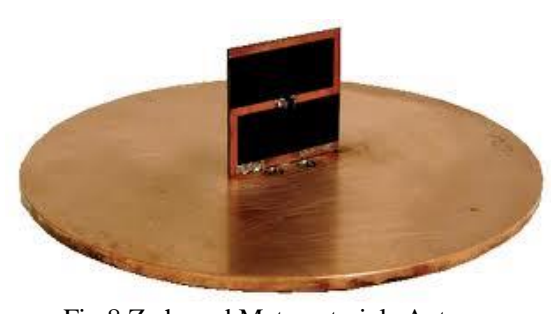

Fig.8 Z-shaped Metamaterials Antenna

The use of artificial materials and surfaces, properly engineered to expand some prescribed antenna features impedance matching, gain bandwidth, efficiency, front-toback ratio, etc [28-29]. Some of metamaterials antennas discussed in literature are below:

\section{1) Zero-Phase-Shift Line Based Antenna}

A perimeter of two operating wavelengths at ultra high frequency (UHF) bands for the near-field RFID applications use a zero-phase-shift segmented loop antennas because it generate larger uniform magnetic field [30-31]. A circularly polarized Omni-directional antenna design with zero-phase- shift line is used for wireless local area network (WLAN) applications [32].

\section{2) Anisotropic High-Permittivity Loaded Antenna}

To construct a multiple-input multiple- output (MIMO) systems dipole antenna is used and is loaded with high permittivity anisotropic metamaterials [33]. To reduce the thickness up to a great extent in arrays of cellular base-station this design concept is used.

\section{3) Mushroom Antenna}

The antenna that designed with array of many mushroom cells is called a mushroom type antenna and is used for the Wi-Fi applications.

\section{4) Gap-Capacitor Loaded Antenna}

This antenna is collection of a number of slotted patches with anti phase and zero-order resonance (ZOR) that introduce a series gap-capacitance at center of the patch and at its radiating edges. This type of antennas is found in L-band radar system applications.

\section{Metamaterials Advantages}

\section{A. Directivity Enhancement}

Metamaterials has inherent property that controls the direction of electromagnetic radiation in order to collect the originating energy in a small angular domain around the normal to the surface [34]. A DNG material enhances the directive properties of an antenna.

\section{B. Bandwidth Enhancement}

Metamaterials antenna increase achieved bandwidth as compared to the conventional patch antenna [35]. This is achieved by use of superstrate of metamateial over conventional antenna or by loading of LHM.

\section{Radiated Power Enhancement}

A small antenna can increase the radiated power through the application of DNG metamaterials [36]. A small dipole antenna enclosed with DNG metamaterials is use to increase the radiated power much more as compared to the conventional antenna.

\section{Beamwidth and side lobes}

The metamaterials antennas decrease the beamwidth and side lobe ratio [37] and thus enhance the directivity and reduce the return loss of antenna.

\section{NUMERICAL TECHNIQUES AND MODELING METHODS OF METAMATERIALS}

There are many complex problems, which can be easily solved by numerical methods only. Various Computational electromagnetic simulators based on Maxwell equations are used to test antennas and wave propagation in complex media. On modeling of metamaterials the researcher should be aware about several special considerations. So, for the efficient 
development of a device, several modeling techniques are used [38]. Some of the methods are discussed below.

\section{A. Finite Difference Time Domain (FDTD) method}

FDTD is an efficient and robust method that used to model a variety of frequency dispersive and non-dispersive materials with electromagnetic wave interaction [39]. It gives straight forward method to model complex periodic structures and commerce with the characteristics of metamaterials over a wide frequency band because it is a time domain solver [40]. The FDTD algorithm gives accuracy in both time and space.

\section{B. Finite-Element Method (FEM)}

To solve the problems of inhomogeneous anisotropic materials and complex structures the finite-element method (FEM) is used [41-42]. It eliminates the problem of spurious solutions by expanding the angular and transverse field components with the node-based scalar and edge-based vector basis functions respectively [43].

\section{Transmission Line Method (TLM)}

There are many numerical methods and theories that describe the properties of different structures of wire metamaterials. The modeling of metamaterials is much important with this method and is also very difficult task. TLM model permits the design of different structures based on conducting thin-wires [44-45].

\section{CONCLUSIONS}

Metamaterials is the new field of research, without any doubt it becomes an extremely exciting research area. The researchers from multiple disciplines are being attracted towards metamaterials because of its unique electromagnetic properties. In this paper, a short review of the history of metamaterials, some of salient features, various types, applications and different modeling methods of metamaterials have been discussed. The metamaterials have resulted in surprising improvements in eelectromagnetic response functions that can offer exciting possibilities of future design of devices, components and salient properties of metamaterials.

\section{REFERENCES}

[1] V. G. Veselago, "The electrodynamics of substances with simultaneously negative values of " $\varepsilon$, and $\mu$ ", Sov. Phys.Usp, Vol. 47, pp.509-514, Jan.-Feb, 1968.

[2] J.P. Pendry, A.J. Holden, D.J. Robbins, W.J. Stewart, IEEE. Trans. Microwave .Theory Technol. 47, 20751999.

[3] J.B. Pendry, A.J. Holden, D.J. Robbins and W.J. Stewart, "Low Frequency Plasmons for Thin-Wire Structure", J. Phys. Condens. Matter, Vol.10, pp. 4785 - 4809, 20 March 1998.

[4] 21. J. Holden, D. J. Robins, and W. J. Stewart, "Magnetism from conductors and enhanced non-linear phenomena," IEEE Trans. Microwave Theory Technol., Vol.47, pp.2075-2084, 1999.

[5] D.R. Smith,W.J. Padila, D.C. Vier, S.C. Nemat-Nasser, S. Schultz, Phys.Rev. Lett. 84, 4184 (2000)

[6] J.B. Pendry, Contemporary Physics 45 191-202, 2004.

[7] D.R. Smith, J.B. Pendry, M.C.K. Wiltshire, Science 305 788-92 (2004).

[8] B.I. Wu, W. Wang, J. Pacheco, X. Chen, T. Grzegorczyk, and J. A. Kong, "A study of using Metamaterial as antenna substrates to enhance gain," Progress in Electromagnetic Research, Vol. 51, pp. 295-328, 2005.

[9] D. R. Smith, W. J. Padilla, D. C.Vier, S.C. Nemat-Nasser, and S. Schultz, "Composite medium with simultaneously negative permeability and permittivity,” Phys. Rev. Lett., 84, pp. 4184-4187, 2000

[10] S. Zhang, W. Fan, N. Panoiu, K. Malloy, R. Osgood, and S. Brueck, Experimental demonstration of near-infrared negative-index metamaterials," Phys. Rev. Lett., vol. 95, no. 13, p.137404, Sep. 2005.

[11] E. Nader and R. W. Ziolkowski, "A positive future for double-negative metamaterials," IEEE Transactions on Microwave Theory and Techniques, vol. 53, pp. 1535-1556, 2005.

[12] R.W. Ziolkowski and A. Kipple, "Causality and double-negative metamaterials,” Phys. Rev. E, vol.68, 026615, Aug. 2003.

[13] Jaggard, D. L.; Mickelson, A. R.; Papas, C. H. (1979). "On electromagnetic waves in chiral media". Applied Physics 18 (2): 211. Bibcode 1979.

[14] Rüdiger Paschotta (2008-18). "Photonic Metamaterials" Encyclopedia of Laser Physics and Technology I \& II Wiley-VCH Verlag. p. 1. Retrieved 2009.

[15] Y. S. Kivshar, Nonlinear and Tunable Metamaterials, in:Metamaterials: Fundamentals and Applications II, edited by M. A. Noginov, N. I Zheludev, A. D. Boardman, andN. Engheta Proc. SPIE 7392, 739217 (2009).

[16] Y. Rahmat Samii, "Metamaterials in Antenna Applications: Classifications, Design and applications." IEEE International Workshop on Antenna Technology Small Antennas and Novel Metamaterials, 1-4, March 6-8, 2006.

[17] Caloz C and Itoh, "Electromagnetic Metamaterials: Transmission Line Theory and Microwave Applications" (New York: Wiley) 2005.

[18] Kurzweil Al "Wrinkled metamaterials for controlling light and sound propagation" 2014-01-28. Retrieved 2014.

[19] Rudykh, S. Boyce, M. C, "Transforming Wave Propagation in Layered Media via Instability-Induced Interfacial Wrinkling". Physical Review Letters 112 (3) 2014.

[20] Sanders, G.H.W. Manz, "A Chip-based microsystems for genomic and proteomic analysis".Trends Anal. Chem. 19, 364-378 2000,

[21] Solinas Toldo, S. Lampel, S. Stilgenbauer, S. Nickolenko, J. Benner, A. Dohner, H. Cremer, T. Lichter, "Matrix-based comparative genomic hybridization: Biochips to screen for genomic imbalances" Genes Chromosomes Cancer 20, 399-407,1997.

[22] Michalet, X. Kapanidis, A.N. Laurence, T. Pinaud, F. Doose, S Pflughoefft, M. Weiss, "The power and prospects of fluorescence microscopies and spectroscopies" Annu. Rev. Biophys. Biomol. Struct. 32, 161-182, 2003.

[23] Webb, S.E.D. Roberts, S.K. Needham, S.R. Tynan, C.J. Rolfe, D.J. Winn, M.D. Clarke, D.T. Barraclough, R. Martin-Fernandez, "Singlemolecule imaging and fluorescence lifetime imaging microscopy show different structures for high- and low-affinity epidermal growth factor receptors in A431 cells" Biophys. J94, 803-819, 2008.

[24] Tao, Hu Landy, Nathan I. Bingham, Christopher M. Zhang, Xin Averitt, Richard D. Padilla, Willie J. "A metamaterial absorber for the terahertz regime: Design, fabrication and characterization" Optics Express 16 (10): 7181-8, 2013.

[25] G. Kiziltas and J. L. Volakis, "Miniature Antenna Designs on Metamaterial Substrates.'

[26] Rajni, Anupma Marwaha, "Metamaterial Antennas: Challenges And Applications"Proceedings of NCCN-11, 4-5 FEBRUARY, 2011.

[27] S.S. Zhong and J.-H. Cui, "Compact circularly polarized microstrip antenna with magnetic substrate," in Proc. IEEE AP-S Int. Symp., San Antonio, TX, vol. 1, pp. 793-796, Jun. 2000.

[28] D. Psychoudakis, Y. H. Koh, J. L. Volakis, and J. H. Halloran, "Design method for aperture-coupled microstrip patch antennas on textured dielectric substrates," IEEE Trans. Antennas Propag., vol. 52, no. 10, pp. 2763-2766, Oct. 2004.

[29] X. Qing and Z. N. Chen, "UHF near-field segmented loop antennas with enlarged interrogation zone," IEEE International Workshop on Antenna Technology (iWAT), pp. 132 - 135, Tucson, USA, March 2012

[30] X. Qing, C. K. Goh, and Z. N. Chen, "A broadband UHF near-field RFID antenna," IEEE Trans. Antennas Propagat., vol. 58, no. 12, pp. 3829-3838, Dec 2010. 
[31] X. Qing and Z. N. Chen, "Horizontally polarized omnidirectional segmented loop antenna," 6th European Conference on Antennas Propagation, pp. 2904-2907, 2012.

[32] P. Y. Lau, Z. N. Chen, and X. Qing, "Gain and bandwidth enhancement of planar antennas using EBG," Asia Pacific Conference on Antennas and Propagation, Singapore, August 2012.

[33] W. Liu, Z. N. Chen, and X. Qing, "Metamaterials-based low-profile broadband mushroom antenna," IEEE Trans Antennas Propagat. accepted for publication in Nov 2013

[34] H. Zhou et al., "Anovel high-directivity microstrip patch antenna based on zero-index metamaterial," IEEE Antennas and Wireless Propagat.Lett., vol. 8, no. 6, pp. 538-541, 2009.

[35] Jui Han Lu, "Bandwidth Enhancement Design of Single layer Slotted Circular MicrostripAntennas" IEEE Transactions on Antenna and propagation, Vol.51, pp 1126-1129 No.5, May 2003.

[36] R.W. Ziolkowski and A. Kipple, "Application of double negative metamaterials to increase the power radiated by electrically small antennas," IEEE Trans. Antennas Propagation, vol.51, pp.2626-2640, Oct. 2003.

[37] Enoch S.et "A metamaterial for directive emission" Phys Rev. Letters 2002.

[38] Arnab Bhattacharya et al, "Modeling Metamaterials 'Metamaterials Development using Electromagnetic Simulation"

[39] Taflove, "Computational Electrodynamics: The Finite-Difference Time-Domain Method" Artech House, Norwood, 1995.

[40] K.S. Yee, "Numerical solution of initial boundary value problems involving Maxwell's equations in isotropic media," IEEE Trans. Antennas Propagaion, Vol. AP-14, No. 3, 302-307, 1966.

[41] A. D. Greenwood and J. M. Jin, "Finite-element analysis of complex axisymmetric radiating structures" IEEE Antenna Propagation, 47, 1260-1266, 1999.

[42] A. D. Greenwood and J. M. Jin, "Computation of the RCS of a complex BOR using FEM with coupled azimuth potentials, PML and Electromagnetics" 19, 147-170, 1999.

[43] F. L. Teixeira and W. C. Chew, "Systematic derivation of anisotropic PML absorbing media in cylindrical and spherical coordinates" IEEE. Microwave Guided Waves, 7, 371-373, 1997.

[44] Hsing Yi Chen, Chieh-Pin Su, "Investigation of return loss of 15 bonding wires fabricated on a test board by FDTD method" IEEE Antennas and Propagation International Symposium, pp. 2313 - 2316 June 2007.

[45] Saenz E., Ikonen P., Gonzalo R., Ederra I., De Maagt P., Tretyakov S., "Modelling and Analysis of Composite Antenna Superstrates Based on Grids of Dipoles and Wires, Antenna Technology: Small and Smart Antennas Metamaterials and Applications" International Workshop (IWAT '07), 21-23 March 2007. 